\title{
High Strain Sustaining, Nitrile Rubber Based, Large-Area, Superhydrophobic, Nanostructured Composite Coatings ${ }^{\text {th }}$
}

\author{
Thomas M. Schutzius ${ }^{\mathrm{a}}$, Manish K. Tiwari ${ }^{\mathrm{b}}$, Ilker S. Bayer ${ }^{\mathrm{c}, \mathrm{d}}$, Constantine \\ M. Megaridis ${ }^{\mathrm{a}, *}$ \\ ${ }^{a}$ Department of Mechanical and Industrial Engineering, University of Illinois at Chicago, \\ Chicago, IL 60607, USA \\ ${ }^{b}$ Department of Mechanical and Process Engineering, Swiss Federal Institute of \\ Technology, Zurich 8092, Switzerland \\ ${ }^{c}$ Center for Biomolecular Nanotechnologies, Smart Materials Platform, Italian Institute \\ of Technology, Arnesano, LE 73010, Italy \\ ${ }^{d}$ Department of Mechanical and Aerospace Engineering, University of Virginia, \\ Charlottesville, VA 22904, USA
}

\begin{abstract}
Elastomeric superhydrophobic nanostructured composite coatings scalable to large areas are prepared by spray casting particle-polymer dispersions. The dispersions consist of nanostructured carbon black particles along with submicrometer-sized poly(tetrafluoroethylene) particles dispersed in nitrile rubber solution in acetone, with the goal to attain superhydrophobicity with minimal content of particle fillers. The coatings are cast on various flexible substrates, which are subsequently stretched uniaxially. Upon drying, water droplet roll-off (sliding) angle measurements are performed to quantify the self-cleaning ability of coated substrates being stretched uniaxially to $30 \%$ strain (coated silicone rubber) and $70 \%$ strain (coated polyester fabric).
\end{abstract}

\footnotetext{
${ }^{\star}$ Preprint of manuscript that appeared in Composites Part A 42 (2011) 979-985.

*email: cmm@uic.edu, phone: (312) 996-3436
} 
The coatings conform to the stretching of the substrates, while maintaining the self-cleaning property throughout this range. Self-cleaning is also maintained for cyclical stretching of coated substrates for strains 0-30\% (coated silicone rubber) and 0-70\% (coated polyester fabric) demonstrating the coatings' functional recovery. Droplet roll-off angles below $10^{\circ}$ reveal good selfcleaning ability for these coatings.

Keywords: A. Nano-structures, A. Thin films, B. Elasticity, B. Wettability

\section{Introduction}

Nitrile rubber, NBR, is a hydrophobic, solution-processable rubber used extensively in the automotive industry (seals, fuel lines, oil hoses, etc.) for its excellent oil, fuel, and heat resistance [1, 2, 3]. Introducing fillers, such as carbon black (CB) and clay, to NBR is well established, with previous works emphasizing mechanical property improvement $[1,2,3,4,5,6,7,8]$. Manipulation of the surface texture with added filler particles is a relatively unexplored feature, which can be used to tune the wettability of the resulting flexible composite coatings. In fact, carefully designed polymer composite coatings with filler particles can even demonstrate superhydrophobicity (static water sessile droplet contact angle over $150^{\circ}$ ) and self-cleaning capabilities (low water droplet roll-off angle or low contact angle hysteresis) $[9,10]$. A hierarchical surface morphology in particular (with micron-tonanoscale roughness) is known to facilitate such high water repellency (lotus effect) $[11,12,13]$. The well-known elastomeric properties of intrinsically hydrophobic nitrile rubber could thus be utilized for the fabrication of flexible, or even stretchable, water repellent composite coatings. Such materials can 
be considered for stretchable substrates (e.g. rubber, textiles) undergoing moderate to intense strain when super-water repellency is required. This approach is expected to work for any elastomeric, hydrophobic, solution processable polymer (e.g. fluoroelastomers).

While there have been abundant previous studies on synthetic superhydrophobic surfaces $[9,10,11,12,13,14,15,16,17,18,19]$, work related to stretchable polymer-based (elastomeric) surfaces is scarce. Zhang et al. [20], produced a polyamide film with a triangular net-like structure that was stretchable and switched between superhydrophobicity and superhydrophilicity (i.e. reversible wetting) depending on its state of strain. Choi et al. [21] presented a method of dip coating fabrics to attach fluorodecyl polyhedral oligomeric silsesquioxane molecules and impart tunable oleophobicity to fabrics subjected to strain; increasing strain resulted in a decrease in oleophobicity. Recently, another group [22] demonstrated the ability to make recycled-rubber-based water repellent surfaces by stamped texture. The literature on simple-to-apply water-repellent coatings for hydrophobizing textiles is quite extensive; see the reviews by Bahners et al. [23] and Gowri et al. [24] for an introduction to currently available methods. For the sake of brevity, we mention only a few relevant techniques. Gao and McCarthy [17] demonstrated a simple dip coating technique for hydrophobizing polyester and microfiber polyester fabrics. Coulson et al. [25, 26] utilized a plasma technique to achieve ultralow surface energy well-adhered polymeric films for creating both hydrophobic and oleophobic surfaces applied on cotton substrates without any solvents (which are required for spray or dip coating applications). Several other authors have also demonstrated relatively sim- 
ple textile coatings that are repellent to both water and low-surface tension liquids (e.g. hexane, hexadecane) [27, 28, 29]. While reversible wetting, rubber-based hydrophobic surfaces, and simple hydrophobic coating application methods for textiles have been demonstrated, works showing the ability to maintain superhydrophobicity (high contact angle) and self-cleaning ability (low roll-off angle) at increasing strains are lacking. In previous studies, the property of liquid repellency was both substrate and strain dependent. In the present work, coatings applied on a variety of flexible substrates maintain superhydrophobicity through a range of strains. We describe a simple, true one-step, spray process based on commodity chemicals applied, either upon manufacture or in a post-processing step, to an existing flexible substrate to impart superhydrophobicity to its surface.

\section{Experimental Section}

The materials used were: PTFE (Sigma Aldrich, $2.15 \mathrm{~g} / \mathrm{cm}^{3}$ density), CB (Cabot, BP2000, $1.9 \mathrm{~g} / \mathrm{cm}^{3}$ density), acetone (99.5\%, Sigma Aldrich), poly(acrylonitrile-co-butadiene) rubber (37-39\% acrylonitrile, Sigma Aldrich, $1 \mathrm{~g} / \mathrm{cm}^{3}$ density), polyester fabric (local fabric store), microscope glass slides, indium foil (Sigma Aldrich, $0.25 \mathrm{~mm}$ thick, 99.99\% metals basis), and silicone rubber $(1 / 2 "$ x 1/16" x 36", McMaster-Carr).

Pieces of NBR were dissolved in acetone at $40^{\circ} \mathrm{C}$ under constant mechanical stirring to obtain a 10 wt. \% solution. PTFE and PTFE-CB suspensions were prepared by dispersing the particles in acetone under sonication for 30 minutes. Upon maintaining a good degree of particle dispersion in acetone, the NBR solution and the suspensions were blended for subsequent 
spray casting. Table 1 describes the composition ranges for the blends used to produce the coatings. Clean microscope glass slides, silicone rubber, and polyester fabric were used as substrates for the coatings. Coatings were spray cast onto the substrates with a single spray application at a fixed distance of $19 \mathrm{~cm}$ using an airbrush atomizer (Paasche VL siphon feed, $0.55 \mathrm{~mm}$ spray nozzle) mounted on an automated industrial dispensing robot (EFD, Ultra TT Series). The airbrush was operated by passing pressurized air through the nozzle to move the particle slurry via siphon feeding; this air stream also acted to augment atomization at the nozzle exit. The pressure drop across the sprayer varied from 2.7-4.1 bar depending on conditions. Each type of filler, and their combination, was incorporated into the dispersion at increasing levels until the dispersion could no longer be sprayed consistently. The coated substrates were dried for 30 minutes at $80^{\circ} \mathrm{C}$ in an oven. The dispersion compositions which yielded superhydrophobic coatings with an optimally low amount of filler particle loading are listed in Table 2. Coatings deposited on flexible substrates were mounted between two linear clamps and stretched using a programmable linear actuator (Velmex). For uniaxial straining, coated polyester fabrics were placed on a stretchable supporting substrate (silicone rubber) in order to facilitate water droplet roll-off angle measurements. The stretching limits used for silicone rubber and polyester fabric were $30 \%$ and $70 \%$ strain, respectively. These limits were chosen based on apparent coating and substrate degradation and not based on loss of superhydrophobicity. Since acetone was the only liquid component of the slurry, the surface tension of the sprayed fluid should be close to that of acetone $\left(22.72 \mathrm{mN} / \mathrm{m}\right.$ at $\left.25^{\circ} \mathrm{C}\right)$. No viscosity measurements were performed for 
these slurries.

A backlit optical image acquisition setup was used to record water droplet images for sessile contact angle (CA) measurements. Water droplet roll-off angle (sliding angle) measurements were performed on a tilting stage (accuracy of 1 degree); the stage was gradually inclined until the droplets rolled off. The water droplet volume used for both $\mathrm{CA}$ and sliding angle measurements was $11.6 \mu \mathrm{l}$. Environmental scanning electron microscope (ESEM) images were obtained using a Philips XL30 ESEM-FEG after all samples were sputter coated with a 5nm-thick layer of gold-palladium. For high-resolution observation of how the composite coating behaves under strain, flexible indium foil substrate was used. Once coated, the indium foil was first stretched to $18 \%$ strain, the upper-limit the indium foil could bear before failing, and was subsequently sputter-coated with $5 \mathrm{~nm}$ gold-palladium before imaging on the ESEM. Transmission electron microscope (TEM) images of the CB filler particles were obtained using a JEOL JEM-2010F. Scanning electron microscope (SEM) images of the PTFE filler particles were obtained in a previous study; the mean diameter of the PTFE particles was measured to be $260 \mathrm{~nm}$ (standard deviation $54.2 \mathrm{~nm}$ ) [10].

\section{Results and Discussion}

Composite coatings containing filler particles allow control of surface roughness. Composite coatings containing both nanometer-scale particles (e.g. CB, size $<100 \mathrm{~nm}$ ) and submicrometer-scale particles (e.g. PTFE, sizes in the range $100-500 \mathrm{~nm})$ generate hierarchical surface roughness, thus

facilitating water repellency. Previous work [10] suggests that while it is 
possible to achieve self-cleaning surfaces by utilizing a single type of filler particle in a composite coating, adding two fillers of different length scales may allow the coating to become self-cleaning at even lower levels of particle loading. Ultimately, utilizing the minimum required amount of filler to attain a self-cleaning surface is of utmost importance for the coatings investigated here, as maximizing the amount of NBR in the final composite coating is essential to its mechanical integrity. Figure 1 shows ESEM images that demonstrate hierarchical micro to nanoscale roughness by utilizing dualscale fillers. Figure 1(a) shows the presence of micro-scale roughness which is attributed to the early dynamic interactions of the fillers, PTFE and CB, with NBR during the spray impact process. Figure 1(b) demonstrates the submicrometer-scale roughness due to the PTFE particles, as well as the nanoscale roughness introduced by the CB particles.

Filler particle addition to a composite coating can affect surface wettability in two different ways. The first is by increasing the surface roughness, and the second is by changing the surface energy, which depends on the wettability of the filler particles themselves (hydrophobic or hydrophilic). Both mechanisms play an important role in affecting the water CA of droplets on coated surfaces. The water CA can be described by either the Wenzel [30] or Cassie-Baxter [31] equations, depending on the state the droplet is in. For heterogeneous surfaces, utilization of these equations requires that the variations in the composite coating's surface texture are small compared to the droplet size, and that they are uniform throughout the surface. If those criteria are satisfied, then increasing liquid repellency as a function of roughness can still be interpreted under the framework of the Wenzel and 
Cassie-Baxter equations [32]. For heterogeneous coatings with well dispersed filler particles and self-similar surface features (see Figure 1) with size well below that of the water droplet (dia. $2.8 \mathrm{~mm}$ ), the increased water repellency of these coatings due to increased surface roughness can be interpreted under the Wenzel and Cassie-Baxter models.

Figure 2 shows water CA measurements on composite coatings with varying filler content and deposited on glass slides. The three curves represent composite coatings prepared using either PTFE particles only, CB particles only, or PTFE and CB particles in a 9:1 PTFE:CB wt. ratio. The figure shows that increasing the concentration of $\mathrm{CB}$ in the dispersion results in a corresponding increase in water $\mathrm{CA}$ for the resulting composite coating. For a $\mathrm{CB}$ concentration of $2 \mathrm{wt}$. \%, a water $\mathrm{CA}$ of $136^{\circ}$ is observed, which is higher than the coatings containing PTFE and PTFE+CB at that same filler concentration. This indicates $\mathrm{CB}$ as an optimal nanofiller when low loading is a factor. On the other hand, PTFE filler particles increase coating roughness on the submicrometer scale, and lower the overall surface energy of the composite. Their ability to be dispersed and sprayed more easily at higher concentrations than their CB counterparts justify PTFE particle use in larger proportion. Figure 2 demonstrates a steady increase in water CA with increasing PTFE content, reaching a maximum of $144^{\circ}$ at 8 wt. $\%$ of dispersion. The combination of PTFE with $\mathrm{CB}(\mathrm{PTFE}+\mathrm{CB})$ also shows a steady increase in CA with filler content, reaching a maximum of $144^{\circ}$ at 7 wt. \% of dispersion. The composite coating with PTFE and CB achieves its maximum water $\mathrm{CA}$ at at a slightly lower level of particle concentration than PTFE alone. 
It has been argued that superhydrophobicity should be characterized by low-CA hysteresis [17], or low sliding angles, as such quantities quantify readiness of water droplet movement (water repellency). Figure 3 shows water droplet roll-off angle (sliding angle) measurements of composite coatings deposited on glass slides for different fillers and their change with varying filler content. The two curves depicted in Figure 3 represent composite coatings prepared using either only PTFE particles or PTFE and $\mathrm{CB}(\mathrm{PTFE}+\mathrm{CB})$ particles in a 9:1 PTFE:CB wt. ratio. The figure demonstrates that adding increasing amounts of PTFE to the dispersion, results in a general reduction in water droplet roll-off angle, which ultimately attains a value $10^{\circ} \pm 2$ at 7 wt. \% of dispersion (composite coating SC2, Table 2). Figure 3 also shows the gradual reduction in roll-off angle with added PTFE + CB fillers, with the composite coating displaying a roll-off angle of $10^{\circ} \pm 3$ at 5 wt. $\%$ of dispersion (composite coating SC1, Table 2), and dropping even lower at higher filler content. While previous works have demonstrated superhydrophobic spray cast coatings with hierarchical structures [10,33], the goal of combining both CB and PTFE here was to achieve superhydrophobicity at the lowest possible level of filler particle concentration in order to maximize the amount of NBR in the final composite coating, thus preserving the coating's mechanical integrity. By utilizing a PTFE $+\mathrm{CB}$ combination, the filler particle concentration necessary for superhydrophobicity was lowered by 2 wt. $\%$ of dispersion over just PTFE alone. It is also interesting to note the relatively large error bar of the roll-off angle for the PTFE+CB case at 4 wt. \%. At this point, the surface transitioned from a sticky Wenzel state to a Cassie-Baxter state, which is characterized by low roll-off angles. The large 
error bar is attributed to the transition between the two wetting states. Since Figure 2 demonstrates increasing $\mathrm{CA}$ for increasing filler content, and since the $\mathrm{CA}$ for PTFE+CB at 3 wt. \% is sufficiently high to guarantee hydrophobicity but the droplet still remains pinned to the surface even when tilted, the droplet must be in a Wenzel state of wetting. On the other hand, at 4 wt. \% no droplet pinning occurs and the droplet must be in a Cassie-Baxter state of wetting. This interpretation is supported by the decrease of the rolloff angles and the corresponding standard deviations with increasing filler concentration, which indicates that the droplets remain in the Cassie-Baxter wetting state.

Repellency to lower surface tension liquids was also investigated to explore the limits of the present composite coatings. A mixture water+IPA (9:1 wt. ratio water:IPA, surface tension $40.42 \mathrm{mN} / \mathrm{m}$ ) [10] was used as a stronger challenge of liquid repellency. Neither coating SC1 or SC2 displayed any droplet sliding behavior, indicating that the water+IPA droplets were in a Wenzel state of wetting. However, the corresponding contact angle values were $126 \pm 3(\mathrm{SC} 1)$ and $104 \pm 3$ (SC2), indicating that the coatings did display mild solvent repellency. Steele et al. [34] showed that in order to produce surfaces repellent to such low surface tension liquids, great care must be taken to contain very low surface energy groups while having the polymer matrix itself develop its own surface texture features (e.g. polymer cells, pores, etc).

For the CB-only filled coatings, the content of the dispersion reached up to 2 wt. \%. No higher CB content was utilized because of clogging of the sprayer during experimentation, corresponding to device limitations. The 
clogging was attributed to the apparent increase in dispersion viscosity and the tendency of the $\mathrm{CB}$ particles to aggregate and clog the sprayer. Previous studies have reported that raising the nanoparticle volume fraction in a suspending liquid can result in disproportionate increases in dynamic viscosity as compared with the pure dispersing fluid [35, 36, 37, 38]. Moreover, undispersed agglomerates, which may persist even after sonic bath treatment [39], can cause increased viscosity in nanoparticle dispersions as well $[36,40]$. Figure 4 confirms the presence of CB aggregates. These aggregates were obtained by drying a small amount of the dispersion placed on a holey carbon film supported by a standard electron microscope grid. Since the limits of breaking up CB aggregates were apparently reached for our sonicator, efforts were made to lower the volume fraction of the nanoparticles (to reduce viscosity) by diluting the dispersion with increased acetone. However, this resulted in large amounts of acetone reaching the surface and causing nonuniform coverage of the substrates by the composite coating.

Figure 5 displays the effect of strain on droplet roll-off angle for coatings SC1 and SC2 deposited on silicone rubber and polyester fabric substrates. It is clear that SC1 preserves the self-cleaning property (roll-off angles $<10^{\circ}$ ) for both silicone rubber and polyester fabric substrates throughout the strain levels investigated. Stretching was ceased at $30 \%$ strain for the silicone rubber substrate because visual crack formation of the coating began to occur beyond that level. Visual crack formation of the coating on the polyester fabric substrate was never noted, but permanent deformation to the substrate began to occur beyond $70 \%$ of strain and for this reason, the straining of the fabric was ceased at that level. It is important to note that in both cases, the 
strain was not ceased due to a loss of superhydrophobicity. Michielsen and Lee [41] utilized Cassie-Baxter theory [31] in its original form (i.e. not simplified to liquid in contact with a flat porous surface) to review the relationship between surface tension and roughness for artificially made superhydrophobic textiles. In that study, the wetting behavior was controlled by varying the geometric structure of the fabric; surface energy was controlled by applying different chemical treatments. In the present study, wetting behavior is influenced solely by the composite coating itself, as roughness is affected by the filler particles, while surface energy is influenced both by the rubber matrix and the particles. If the geometric structure of the underlying textile did significantly affect the wettability of the surface, then there would be a measurable difference in wettability between a flat coated substrate (e.g. glass slide, silicone rubber) and a textured coated substrate (e.g. textile). Figure 5 shows that the coating when on a flat substrate (silicone rubber) has similar droplet roll-off angles as when on the fabric for varying stretched states. This indicates that the fabric's surface texture (i.e. roughness, which is also expected to change with stretching) is not contributing to superhydrophobicity. One of the goals in developing the present coating system was to be able to apply it to a variety of flexible substrates without relying on each substrate's inherent roughness to create superhydrophobicity.

Figure 6 shows roll-off angles for coatings SC1 and SC2 deposited on fabric and silicone rubber substrates and their values during cycling at their corresponding strain limits. SC1 remains self-cleaning on both silicone rubber and polyester fabric beyond the four strain cycles. Coating SC2 with roll-off angles between $10^{\circ}$ and $20^{\circ}$ is not quite self-cleaning, but maintains 
its superhydrophobic property throughout and beyond the four strain cycles. It is argued that the higher strains achievable with the coated fabric (without visual crack formation) were due to the fact that the coating conformed to the fibers of the fabric rather than plugging its pores. A conformal coating is supported by the ESEM images of Figure 7 where the coating clearly conforms to the bulk fibers. Choi et al. [21] also demonstrated a conformal style coating for their strained polyester fabrics with tunable oleophobicity. In Fig. 6 , we present only a few cycles of straining to show that superhydrophobicity is maintained after repeated straining and contracting. Abrupt failure of the coating was not observed after the four strain-contraction cycles; moreover, loss of superhydrophobicity was not observed even after one-hundred straincontraction cycles, although the coating did begin to show slow mechanical degradation with increasing number of cycles.

Figures 8(a)-(c) present ESEM images of coating SC1 deposited on stretchable indium foil. Once coated, the indium foil was strained to $18 \%$. Figure 8(a) shows the presence of crack-like defects, which are randomly distributed on the coating, but have a preferred direction of propagation perpendicular to the applied tension [42]. During the formation of the crack-like structures, nanofibers formed from the rubber binder were seen to bridge the microcracks; (c). The formation of small fibers is common in fracture of glassy thermoplastics, where these fibers are referred to as fibrils, and the effect associated with their formation is referred to as crazing [43]. As seen in (b), the fibril orientation is perpendicular to the direction of crack propagation; the presence of fibrils is also quite homogeneous with respect to the depth of the crack; (c). The average diameter of the observed fibrils is $70 \pm 33 \mathrm{~nm}$, which 
is of the same order with previously observed polymer fibrils (5-30nm dia.) [43]. Crazing is known to increase energy dissipation and fracture toughness, due to the ability of the fibrils to support some load. While it is unclear whether the fibrils themselves increase the fracture toughness of the present composites, these fibrils play a positive roll in keeping the coating intact and delaying macroscopic crack formation. It is also interesting to note the shape of the fibrils themselves, which appear to be cylindrical but also decorated with 'pearls'. Periodic beads seem to form along the entire length of the individual fibrils, as previously observed in nanofibers electrospun from polymer solutions of low viscoelasticity [44, 45].

Figures 8(d)-(f) present ESEM images of coating SC1 applied on stretchable silicone rubber. Once coated, this substrate was stretched to $30 \%$ strain and then allowed to relax. Figures 8(d)-(f) display images of the coating surface at its relaxed state (i.e. $0 \%$ strain) and reveal the presence of microcracks. Prior to crack formation, the composite coating appears to have formed fibrils, which broke probably under excessive strain. Remnants of broken fibrils in Figure 8(f) indicate their earlier presence under reduced strain. So while superhydrophobicity of the composite coating on silicone rubber persists for stretching between 0-30\% strain, coating fracture and thus mechanical failure occurs at strains below 30\%. This low level of strain required for the composite coating to fracture, when compared to other NBR composites, is attributed to the high level of particle filler concentration required for superhydrophobicity (3.3:1 filler particle:NBR mass fraction). It should be noted, though, that flaking off of the coating, as previously observed for other superhydrophobic surfaces undergoing significant stretching, 
is not observed for the levels of strain investigated here.

\section{Conclusion}

High strain sustaining (stretchable), superhydrophobic, nanostructured, composite coatings have been synthesized by solution-based processing of nitrile rubber and two separate particle fillers, nanoscale carbon black and submicron-scale poly(tetrafluoroethylene). The coatings were optimized in terms of minimizing particle filler mass content. The ability of these coatings to conform to high strains of the underlying flexible substrate was demonstrated. Superhydrophobicity of the coatings was demonstrated for uniaxial stretching up to $30 \%$ strain on silicone rubber, $70 \%$ strain on polyester fabric, and for cyclic stretching on these two substrates. Water droplet roll-off angles were low enough in some cases to deem these coatings self-cleaning even under extreme strain. Electron microscope visualization of coatings under strain revealed that fibrils form as a result of crazing, and play a role in de-

laying the true fracture of the composite at high strains. The present results are important as they offer a facile method to synthesize large-area, flexible, conforming, super-repellent coatings with commercially available bulk ingredients.

\section{Appendix}

Please refer to the supplementary material for a video demonstrating the spraying process as well as the water repellency of the coating at both stretched and contracted states. 


\section{References}

\section{References}

[1] B. Rodgers, W. H. Waddell, Rubber Compounding, 3rd Edition, Vol. 11 of Encyclopedia of Polymer Science and Technology, John Wiley \& Sons, Hoboken, NJ, 2004, pp. 623-670.

[2] G. R. Hamed, Engineering with Rubber: How to design Rubber Components, 2nd Edition, Hanser Gardner, Cincinnati, OH, 2001, Ch. 2, pp. $16-34$.

[3] R. C. Klingender, Handbook of Specialty Elastomers, Taylor \& Francis, Boca Raton, FL, 2008, Ch. 2, pp. 39-92.

[4] M. Han, H. Kim, E. Kim, Nanocomposites prepared from acrylonitrile butadiene rubber and organically modified montmorillonite with vinyl groups, Nanotechnology 17 (2006) 403-409.

[5] J. T. Kim, D. Y. Lee, T. S. Oh, D. H. Lee, Characteristics of nitrilebutadiene rubber layered silicate nanocomposites with silane coupling agent, Journal of Applied Polymer Science 89 (10) (2003) 2633-2640.

[6] C. Nah, H. J. Ryu, S. H. Han, J. M. Rhee, M. H. Lee, Fracture behaviour of acrylonitrile-butadiene rubber/clay nanocomposite, Polymer International 50 (11) (2001) 1265-1268.

[7] C. Nah, H. J. Ryu, W. D. Kim, Y. W. Chang, Preparation and properties of acrylonitrile-butadiene copolymer hybrid nanocomposites with organoclays, Polymer International 52 (8) (2003) 1359-1364. 
[8] S. Sadhu, A. K. Bhowmick, Preparation and properties of nanocomposites based on acrylonitrile-butadiene rubber, styrene-butadiene rubber, and polybutadiene rubber, Journal of Polymer Science Part B: Polymer Physics 42 (9) (2004) 1573-1585.

[9] I. S. Bayer, M. K. Tiwari, C. M. Megaridis, Biocompatible poly(vinylidene fluoride)/cyanoacrylate composite coatings with tunable hydrophobicity and bonding strength, Applied Physics Letters 93 (2008) 173902.

[10] M. K. Tiwari, I. S. Bayer, G. M. Jursich, T. M. Schutzius, C. M. Megaridis, Highly liquid-repellent, large-area, nanostructured poly (vinylidene fluoride)/poly (ethyl 2-cyanoacrylate) composite coatings: Particle filler effects, ACS Applied Materials \& Interfaces 2 (4) (2010) $1114-1119$.

[11] L. Gao, T. McCarthy, The lotus effect explained: two reasons why two length scales of topography are important, Langmuir 22 (7) (2006) 29662967.

[12] D. Quere, Non-sticking drops, Reports on Progress in Physics 68 (2005) 2495-2532.

[13] M. Ma, R. M. Hill, Superhydrophobic surfaces, Current Opinion in Colloid \& Interface Science 11 (4) (2006) 193-202.

[14] I. S. Bayer, A. Steele, P. J. Martorana, E. Loth, L. Miller, Superhydrophobic cellulose-based bionanocomposite films from pickering emulsions, Applied Physics Letters 94 (16) (2009) 163902. 
[15] I. S. Bayer, A. Steele, P. J. Martorana, E. Loth, Fabrication of superhydrophobic polyurethane/organoclay nano-structured composites from cyclomethicone-in-water emulsions, Applied Surface Science 257 (2010) 823-826.

[16] R. Furstner, W. Barthlott, C. Neinhuis, P. Walzel, Wetting and selfcleaning properties of artificial superhydrophobic surfaces, Langmuir 21 (3) (2005) 956-961.

[17] L. Gao, T. McCarthy, Artificial lotus leaf prepared using a 1945 patent and a commercial textile, Langmuir 22 (14) (2006) 5998-6000.

[18] S. H. Kim, Fabrication of superhydrophobic surfaces, Journal of Adhesion Science and Technology 22 (3-4) (2008) 235-250.

[19] A. Tuteja, W. Choi, G. H. McKinley, R. E. Cohen, M. F. Rubner, Design parameters for superhydrophobicity and superoleophobicity, MRS Bulletin 33 (8) (2008) 752-758.

[20] J. Zhang, X. Lu, W. Huang, Y. Han, Reversible superhydrophobicity to superhydrophilicity transition by extending and unloading an elastic polyamide film, Macromolecular Rapid Communications 26 (6) (2005) 477-480.

[21] W. Choi, A. Tuteja, S. Chhatre, J. M. Mabry, R. E. Cohen, G. H. McKinley, Fabrics with tunable oleophobicity, Advanced Materials 21 (21) (2009) 2190-2195.

[22] E. Bormashenko, V. Goldshtein, R. Barayev, T. Stein, G. Whyman, R. Pogreb, Z. Barkay, D. Aurbach, Robust method of manufacturing 
rubber waste-based water repellent surfaces, Polymers for Advanced Technologies 20 (7) (2009) 650-653.

[23] T. Bahners, T. Textor, K. Opwis, E. Schollmeyer, Recent approaches to highly hydrophobic textile surfaces, Journal of Adhesion Science and Technology 22 (2008) 285-309.

[24] S. Gowri, L. Almeida, T. Amorim, N. Carneiro, A. Pedro Souto, M. Fátima Esteves, Polymer nanocomposites for multifunctional finishing of textiles-a review, Textile Research Journal 80 (13) (2010) 12901306.

[25] S. R. Coulson, I. S. Woodward, J. P. S. Badyal, S. A. Brewer, C. Willis, Plasmachemical functionalization of solid surfaces with low surface energy perfluorocarbon chains, Langmuir 16 (15) (2000) 6287-6293.

[26] S. R. Coulson, I. S. Woodward, J. P. S. Badyal, S. A. Brewer, C. Willis, Ultralow surface energy plasma polymer films, Chemistry of Materials 12 (7) (2000) 2031-2038.

[27] S. Brewer, C. Willis, Structure and oil repellency: Textiles with liquid repellency to hexane, Applied Surface Science 254 (20) (2008) 64506454.

[28] H. Hoefnagels, D. Wu, W. Ming, Biomimetic superhydrophobic and highly oleophobic cotton textiles, Langmuir 23 (26) (2007) 13158-13163.

[29] B. Leng, Z. Shao, W. Ming, Superoleophobic cotton textiles, Langmuir 25 (4) (2009) 2456-2460. 
[30] R. N. Wenzel, Resistance of solid surfaces to wetting by water, Industrial \& Engineering Chemistry 28 (8) (1936) 988-994.

[31] A. Cassie, S. Baxter, Wettability of porous surfaces, Transactions of the Faraday Society 40 (1944) 546-551.

[32] M. Nosonovsky, On the range of applicability of the Wenzel and Cassie equations, Langmuir 23 (19) (2007) 9919-9920.

[33] P. Manoudis, I. Karapanagiotis, A. Tsakalof, I. Zuburtikudis, C. Panayiotou, Superhydrophobic composite films produced on various substrates, Langmuir 24 (19) (2008) 11225-11232.

[34] A. Steele, I. Bayer, E. Loth, Inherently superoleophobic nanocomposite coatings by spray atomization, Nano Letters 9 (1) (2009) 501-505.

[35] B. Pak, Y. Cho, Hydrodynamic and heat transfer study of dispersed fluids with submicron metallic oxide particles, Experimental Heat Transfer 11 (2) (1998) 151-170.

[36] X. Wang, X. Xu, S. Choi, Thermal conductivity of nanoparticle-fluid mixture, Journal of Thermophysics and Heat Transfer 13 (4) (1999) $474-480$.

[37] C. Nguyen, F. Desgranges, G. Roy, N. Galanis, T. Mare, S. Boucher, H. Angue Mintsa, Temperature and particle-size dependent viscosity data for water-based nanofluids-hysteresis phenomenon, International Journal of Heat and Fluid Flow 28 (6) (2007) 1492-1506. 
[38] Z. Jia-Fei, L. Zhong-Yang, N. Ming-Jiang, C. Ke-Fa, Dependence of nanofluid viscosity on particle size and $\mathrm{pH}$ value, Chinese Physics Letters 26 (6) (2009) 066202.

[39] Y. Hwang, J. Lee, Y. Jeong, S. Cheong, Y. Ahn, S. Kim, Production and dispersion stability of nanoparticles in nanofluids, Powder Technology 186 (2) (2008) 145-153.

[40] R. Prasher, D. Song, J. Wang, P. Phelan, Measurements of nanofluid viscosity and its implications for thermal applications, Applied Physics Letters 89 (2006) 133108.

[41] S. Michielsen, H. Lee, Design of a superhydrophobic surface using woven structures, Langmuir 23 (11) (2007) 6004-6010.

[42] D. Gross, T. Seelig, Fracture Mechanics: With an Introduction to Micromechanics, Mechanical Engineering Series, Springer, New York, NY, 2006, Ch. 3, p. 56.

[43] E. Kramer, Microscopic and molecular fundamentals of crazing, Vol. 5253 of Advances in Polymer Science, Springer, New York, NY, 1983, pp. $1-56$.

[44] Y. Liu, J. He, J. Yu, H. Zeng, Controlling numbers and sizes of beads in electrospun nanofibers, Polymer International 57 (4) (2008) 632-636.

[45] D. H. Reneker, A. L. Yarin, Electrospinning jets and polymer nanofibers, Polymer 49 (2008) 2387-2425. 


\section{Tables \& Figures}

Table 1: Composition Range of NBR/Particle Dispersions

\begin{tabular}{ll}
\hline \multicolumn{1}{c}{ Ingredient } & Concentration (wt. \%) \\
\hline NBR & 1.5 \\
filler particles & $1.0-9.0$ \\
acetone & $97.5-89.5$ (balance)
\end{tabular}

Table 2: Dispersion Compositions for Elastomeric Superhydrophobic Coatings with Minimal Particle Content

\begin{tabular}{lll}
\hline Ingredient & SC1 (wt. \%) & SC2 (wt. \%) \\
\hline NBR & 1.5 & 1.5 \\
CB & 0.5 & 0.0 \\
PTFE & 4.5 & 7.0 \\
acetone & 93.5 & 91.5
\end{tabular}



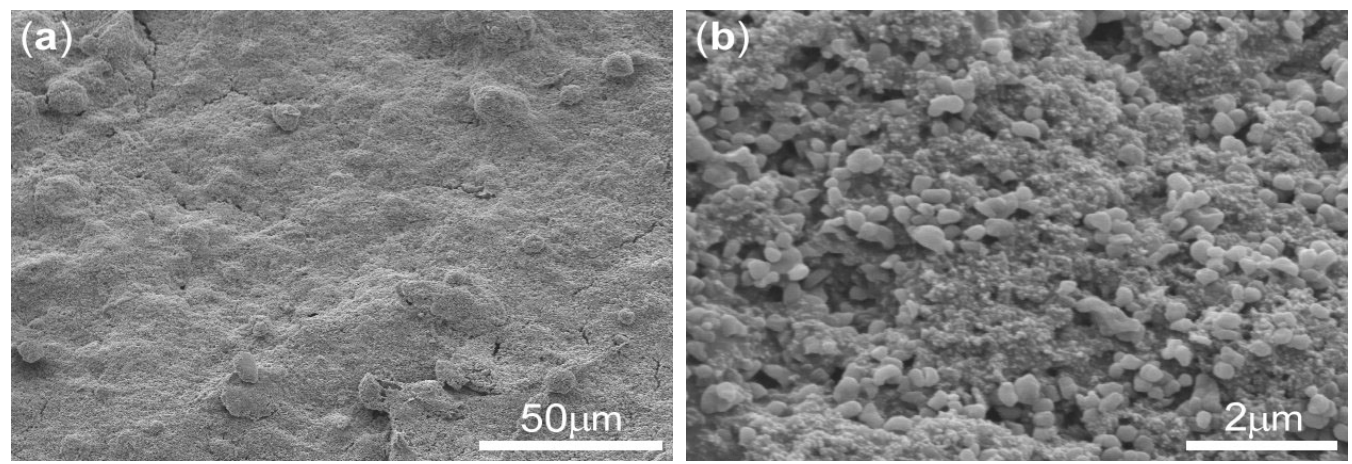

Figure 1: ESEM images of composite coating SC1, deposited on indium foil, unstrained, demonstrating hierarchical roughness by dual filler particle addition for (a) low magnification and (b) higher magnification.

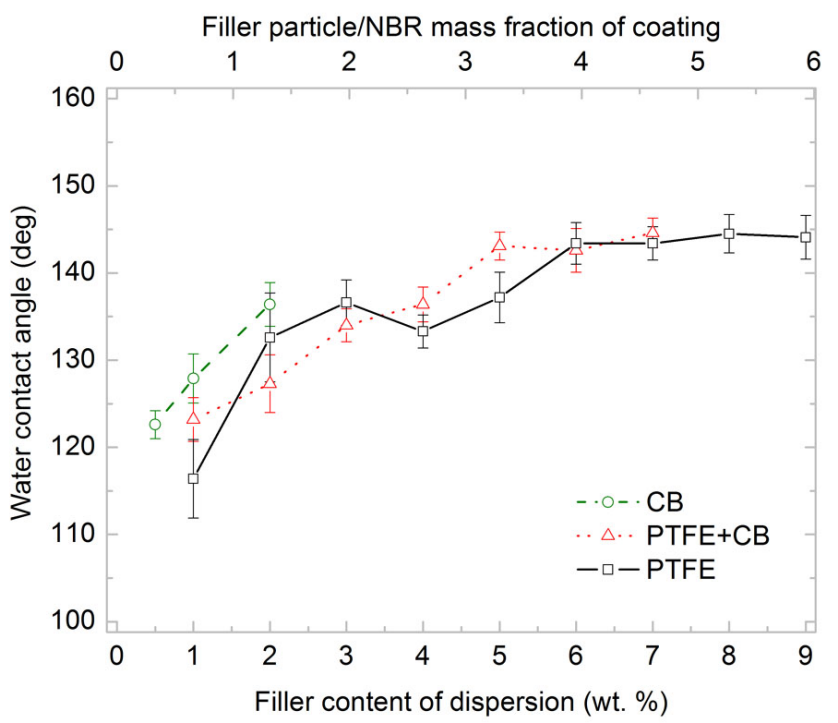

Figure 2: Sessile water droplet CA variation as a function of filler particle content for coatings with different fillers. The PTFE+CB case maintains a filler particle wt. ratio of 9:1 PTFE:CB. The coatings were deposited on glass slides. Each data point represents 10 water CA measurements. 


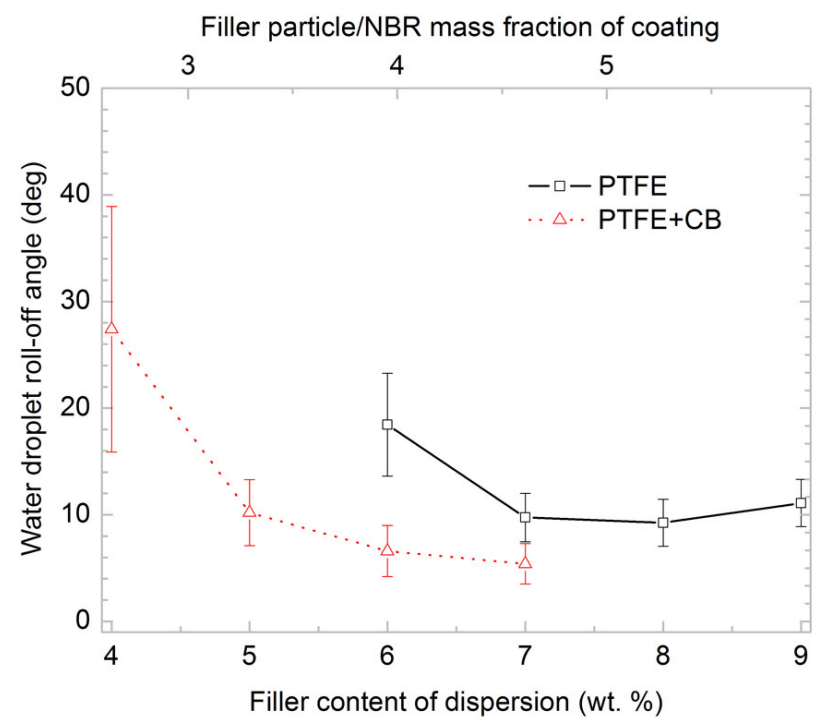

Figure 3: Water droplet roll-off (sliding) angle as a function of filler particle dispersion content for coatings with different fillers. The coatings were deposited on glass slides. 40 measurements were averaged for each data point. For filler dispersion concentrations less than 4 wt. \% PTFE+CB and 6 wt. \% PTFE, water droplets remained pinned to the coating's surface even when inverted. 


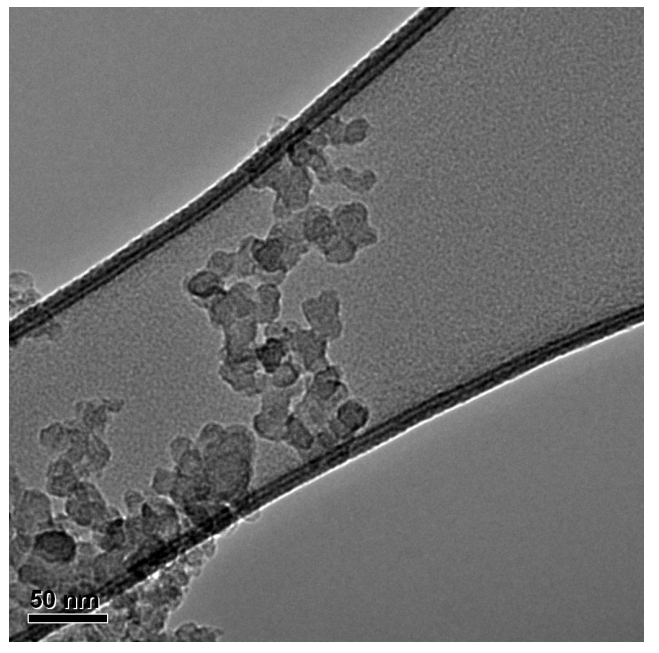

Figure 4: TEM image showing the morphology of the CB filler particles used in this work. Image analysis showed that the mean diameter of the CB primary units is $21 \mathrm{~nm}$ (standard deviation $3 \mathrm{~nm}$ ). 


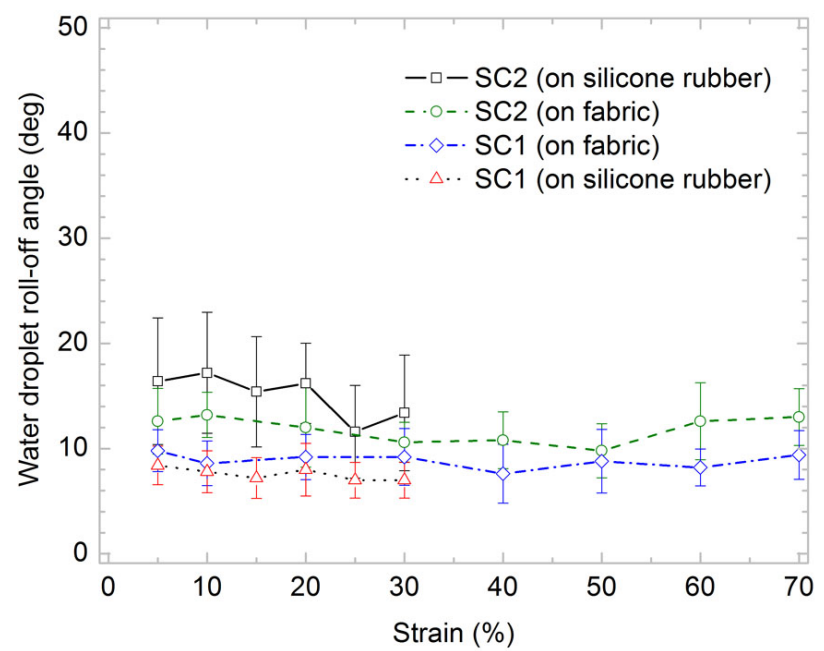

Figure 5: Water droplet roll-off angles as a function of strain for coatings deposited on silicone rubber and polyester fabric. 10 roll-off angle measurements were averaged for each data point. Uncoated silicone rubber did not allow droplet roll-off even when inverted. The uncoated fabric showed inconsistent roll-off angles in the range of $30^{\circ}$ to $90^{\circ}$. 


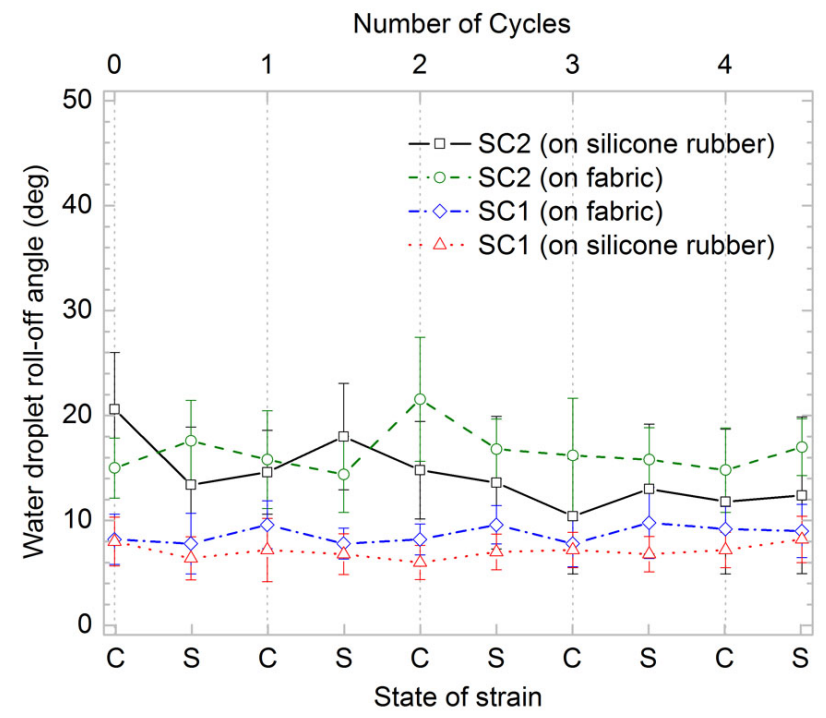

Figure 6: Water droplet roll-off angles for coatings deposited on silicone rubber and polyester fabric at both stretched $(\mathrm{S})$ and contracted $(\mathrm{C})$ conditions. Coated silicone rubber substrates were cyclically stretched to $30 \%$ strain, while coated fabric substrates were cyclically stretched to $70 \%$ strain. 10 measurements were averaged to obtain each data point. 

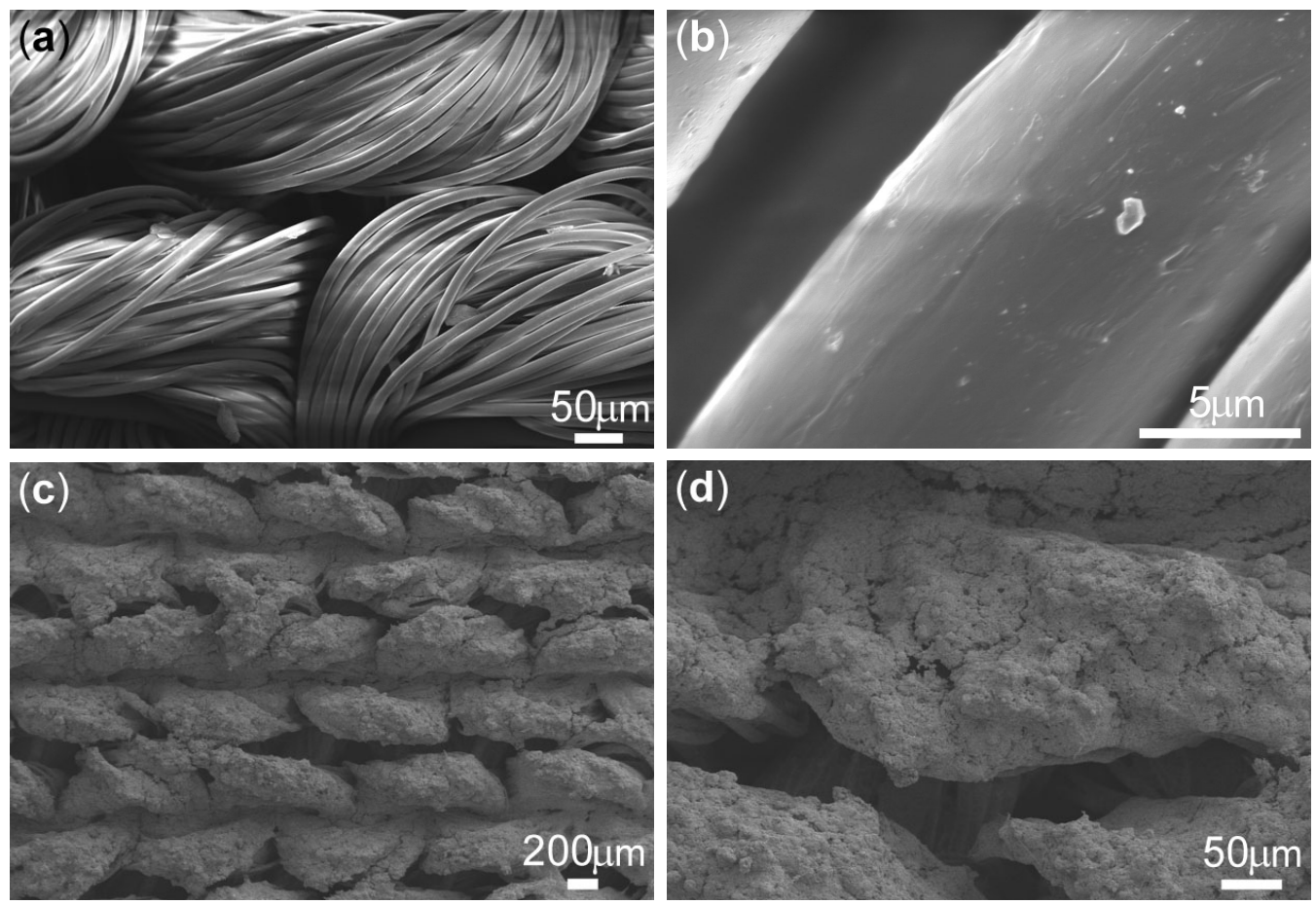

Figure 7: ESEM images of the uncoated polyester fabric at (a) lowmagnification, and (b) higher magnification. ESEM images of composite coating SC1, deposited on polyester fabric, unstrained, demonstrating conformal coating of the fabric fibers at (c) low magnification, and (d) higher magnification. 

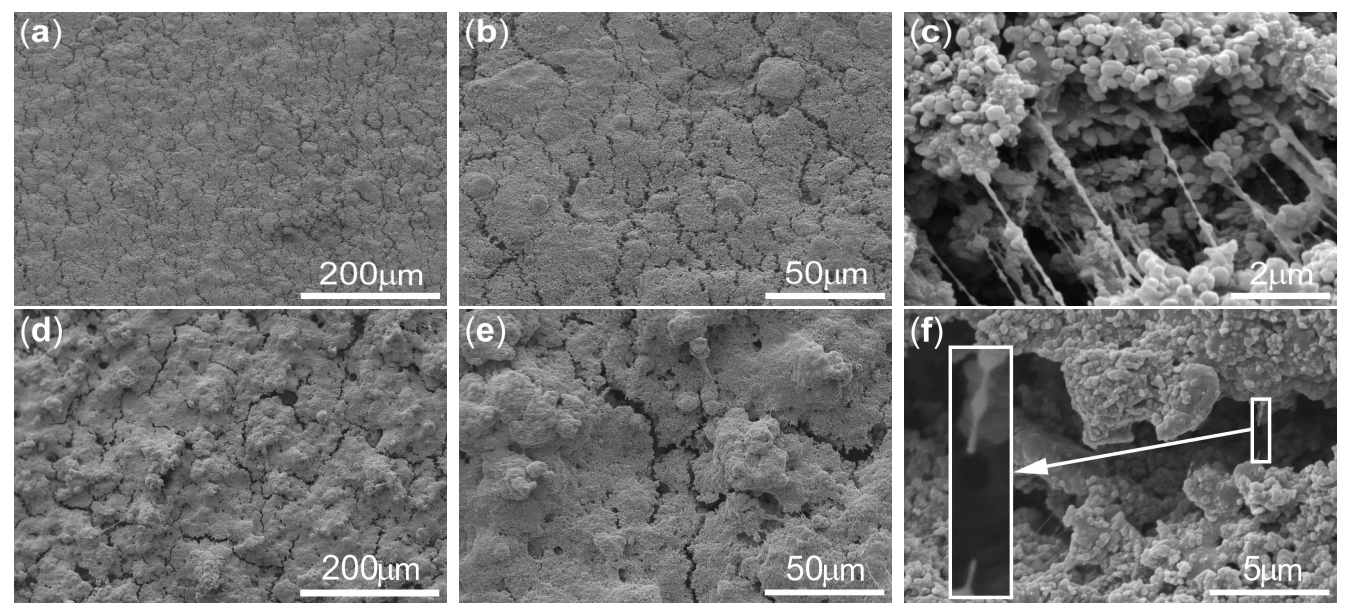

Figure 8: (a)-(c) ESEM images of composite coating SC1 (deposited on indium foil, strained to $18 \%$ ) at different magnifications increasing from left to right showing crazing. (d)-(f) ESEM images of composite coating SC1 deposited on silicone rubber relaxed after stretching to $30 \%$ strain. The inset in (f) displays a magnified image of broken fibrils. 